\title{
Design of a High-Bandwidth Y-Shaped Photonic Crystal Power Splitter for TE Modes
}

\author{
Mohammad Danaie*, Ruhallah Nasiri-Far, and Abbas Dideban \\ Faculty of Electrical and Computer Engineering, Semnan University, Semnan, Iran \\ ${ }^{*}$ Corresponding Author Email: danaie@,semnan.ac.ir
}

Received: Nov. 22, 2016, Revised: Apr. 4, 2017, Accepted: May. 6, 2017, Available Online: Oct. 28, 2017

\begin{abstract}
In this paper, a Y-shaped power splitter based on a two dimensional photonic crystal (PhC) for TE modes is designed and optimized. A triangular lattice of air holes is used for Y-shaped power divider. For analyzing these structures, plane wave expansion (PWE) and finite difference time domain (FDTD) methods are used. The simulation results show that more than $98 \%$ of the input power is transmitted to the outputs and the structure has just less than $2 \%$ reflected power. According to the simulation results this structure is suitable for high bandwidth optical integrated circuit at the $1550 \mathrm{~nm}$ wavelength.
\end{abstract}

KEYWORDS: power splitter, photonic crystal, FDTD, Y-junction, optical power divider.

\section{I.INTRODUCTION}

One of the new topics in the field of electronics and telecommunications is the development of optical technology for high bandwidth devices. Due to the rapid expansion of technology, the need to transfer more data has become crucial. Nowadays, one of the most important media in data transfer is the optical fiber. It is the subject of industrial research around the world. Until now, good progress has been achieved in this area and every day their quality is improved. Photonic crystal (PhC) technology is one of the new techniques in the manufacture of optical fibers. They are compatible with standard optical communication technologies [1], [2] and with electronic integrated circuit fabrication process. Today, due to the advances made in the field of telecommunications, information sharing with high speed is essential. Electronic systems are not able to meet these requirements anymore. The wide bandwidth of optic fibers and optical communication networks made optical devices more attractive for researchers. Among the structures used to design optical devices are photonic crystals. Photonic crystals exhibit a special frequency range [3], known as optical bandgap, where no electromagnetic wave in this frequency range is allowed to propagate in the crystal structure [4]-[7]. It gives them unique characteristics which can be employed for designing many optical devices

The easiest way to create a $\mathrm{PhC}$ is to create holes in a dielectric slab. If a row of holes is removed, a waveguide can be created and as a result a local mode may be able to propagate inside. Due to the existence of the bandgap, light can only move along the path of holes that are removed. Therefore, it acts as an optical waveguide and light emission is limited to a specific path. This optical waveguide has considerable potential for use in integrated optical devices, including: optical filters, optical switches, bends, multiplexers, power splitters, etc. Power splitters are among the main components in optical communication systems that are used for many applications. Power splitters have different types such as directional-coupler-based [8], Yshaped [9] and T-shaped [10]. Recently, photonic crystals have been used to design power splitters in optical integrated circuits [11]. Power splitters can be designed with different number of outputs. The input optical signal of the splitter, due to its design is 
divided between the outputs, but some of that power may return to the input as a reflected power. Many have tried to optimize the structures of $\mathrm{PhC}$ power splitters. Among the most recent ones, a topology has been proposed by Nozhat and Granpayeh. It consists of a Y-shaped line defect. They used an effective numerical method based on FDTD scheme to obtain and improve the parameters of their structure [12]. Another Y-shaped power splitter was investigated by Gaffari et al. [13]. According to it, bi-periodic structures can show much better performances compared to their conventional counterparts.

Yang succeeded to design a Y-shaped power splitter with ultra-low loss outputs and a 162 $\mathrm{nm}$ bandwidth. The design is suitable for TEpolarized light and is designed based on a $\mathrm{PhC}$ slab in a silicon-on-insulator (SOI) material [14]. A polarization power splitter is designed by Li that has two outputs, one of its outputs was for TE and another output for TM polarization which can be used for high speed applications [15]. Then, Lin designed a new Y-type polarization power splitter in a squarelattice $\mathrm{PhC}$ with a wide wavelength and low loss [16]. After that, Huang presented another structure for polarization beam splitter. $\mathrm{He}$ improved the problems of polarization error caused by the insufficient performance of extinction ratio in the process of transmission [17]. In the year 2012, Badaoui presented a new Y-shaped power splitter topology for TE polarized light. It used $\mathrm{InP} / \mathrm{GaInAsP} / \mathrm{InP}$ with a triangular array of holes. The total power transmission of both output ports was about $75 \%$ [18]. Another design for Y-junction splitter has claimed to overcome some of the difficulties such as mode mismatch, bandwidth and bending region transmission and challenges. This structure can be used in communication systems and has the ability to be integrated [19]. One photonic crystal structure for wide-band Y-splitter for TE modes with a triangular lattice of air holes etched in a GaAs slab also is presented in [20]. It has less complexity and about $100 \mathrm{~nm}$ bandwidth.
PhC power splitters are designed not only with two output ports [21]-[23] but also with three [25], [26], four [27]-[29] and even eight output ports [30]. A Y-shaped beam splitter has been designed using composite where the power ratio of each output can be adjusted by changing the location of the input beam [31]. In the year 2013, a slow-light PhC Y-junction was presented that which had the potential to be used also as a Mach-Zehnder interferometer [32]. After that in 2014, a $\mathrm{PhC}$ power splitter was designed that consisted of two parallel coupled-cavity waveguides placed in proximity. That structure can be used for splitting high-speed optical signals in integrated optical circuits [33]. In this paper, a Y-shaped structure is presented. It provides a high bandwidth and constant power ratio. The rest of the paper is organized as follows: In Section 2 we describe parameters and simulation methods used in this paper. Section 3 explains the $\mathrm{Y}$-shaped design proposed to obtain more beam splitting efficiency and optimization method for obtaining different powers in the outputs. Section 4 presents the simulation results regarding the proposed structures, and the final section is devoted to the conclusions.

\section{NUMERICAL METHOD}

In this paper, GaAs is used as the substrate. The refractive index of GaAs is about 3.4 for wavelengths around $1550 \mathrm{~nm}$. In our designs a PhC slab with a triangular pattern of circular holes is used for Y-shaped power splitter structures. Assuming that " $a$ " is the lattice constant of the structure. The radius of the holes in this structure is $0.3 \mathrm{a}$. The slab thickness of triangular pattern is about $0.6 \mathrm{a}$ and the $\mathrm{PhC}$ is assumed to have an air cladding [20]. Finite Difference Time Domain (FDTD) method and Plane Wave Expansion (PWE) are used to analyze the structures. A bandgap, in two dimensional slabs with finite height, is defined by the range of frequencies in which no "guided mode" exists. The bandgap will not be a complete bandgap, because there are leaky modes at those frequencies. 


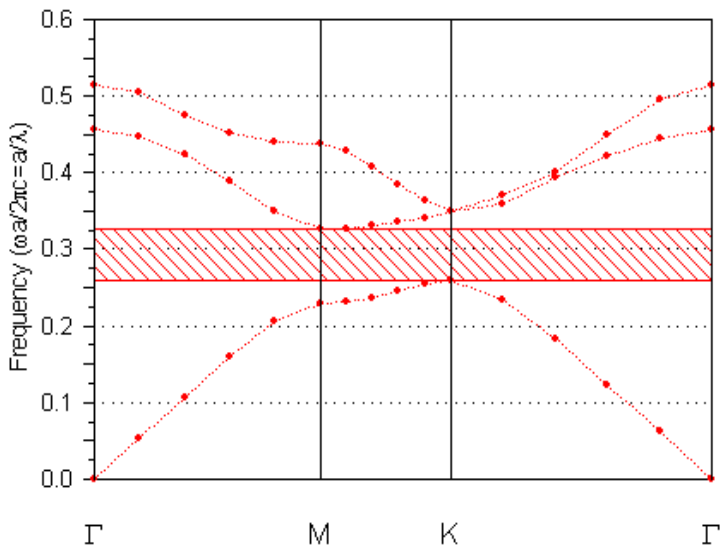

Fig. 1. Simulated photonic band structure of 2D triangular lattice PhCs.

The band diagram, for the $\mathrm{PhC}$ slab used in this paper is shown in Fig. 1. As seen this type of $\mathrm{PhC}$ has a bandgap for TE-like modes. This diagram is obtained by two dimensional plane wave expansion (PWE) method using an effective index equal to 2.76 .

\section{DESCRIPTION OF Y-SHAPED POWER SPLITTER}

Scientists have investigated photonic crystals consisting of dielectric rods in air and/or array of holes in a dielectric slab. They studied on T-junctions, Y-junctions and waveguide branches [34]-[36]. The rod-type $\mathrm{PhC}$ has a poor vertical confinement and its implementation for most of optical devices is difficult. In slab waveguide structures, the air holes are etched into a dielectric such as silicon [37], GaAs/AlGaAs hetero-structure [38] and a semiconductor membrane [39]-[41].

The problem of creating holes in dielectric is that the single-defect $\mathrm{PhC}$ waveguide becomes multi-mode [41]-[43]. It results in modemixing problem in junction of waveguides. It creates a mismatch in the fields between input and output and makes larger reflections. While the researchers have studied about bends and straight waveguides, the junction problems have only recently received attention [44][47]. The Y-splitter design consists of three waveguides which are connected together. Conventional $\mathrm{Y}$-splitters have strong reflections and a narrow bandwidth. Because of strong reflection, the input power that is transmitted to the output ports is only $20 \%$ [48]. An alternate design based on a triple line defect waveguide has designed by [44]. They presented a structure that has $25 \mathrm{~nm}$ bandwidth and $45 \%$ power transmission by adding an additional hole at the junction.

\section{Proposed Structure and SiMULATION RESULTS}

The PhC Y-junction structure is defined by an array of air holes in substrate with refractive index of 3.4 (GaAs). The regular holes are placed in a triangular lattice and have a radius $\mathrm{r}=0.3 \mathrm{a}$, where lattice constant $\mathrm{a}=430 \mathrm{~nm}$. The $\mathrm{PhC} \mathrm{Y}$-junction is formed by the intersection of three $\mathrm{PhC}$ waveguides at $120^{\circ}$ as shown in Fig. 2. The output channels of $Y$ splitter are parallel to the input channel and have a $60^{\circ}$ bend.

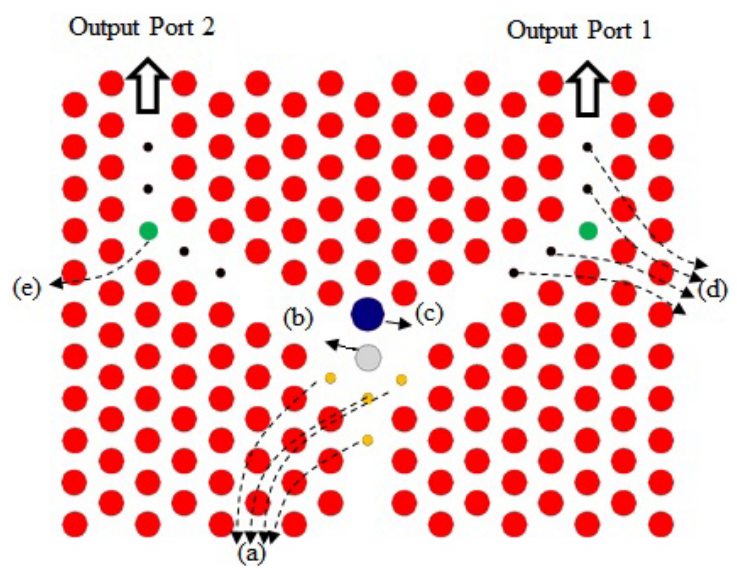

Fig. 2. The proposed Y-shaped photonic crystal topology for TE polarized incident light. The lattice defects have a radius equal to $0.3 a$, while the radii of the labeled holes are: $r_{a}=0.12 a, r_{b}=0.31 a$, $r_{c}=0.4 a, r_{e}=0.19 a$, and $r_{d}=0.11 a$.

The $120^{\circ}$ junction and $60^{\circ}$ bend represent severe discontinuities in the $\mathrm{PhC}$ waveguides and are potential regions in which the singlemode operation might suffer from large transmission losses. Therefore, the discontinuities in these regions are carefully designed. The band diagram of the W1 waveguide used in Y-shaped power splitter is shown in Fig. 3. The radius of the slab holes is 
0.3a. The radius of specified holes is: $r_{a}=0.12 a, r_{b}=0.31 a, r_{c}=0.4 a, r_{e}=0.19 a$, and $r_{d}=0.11 a$. This structure has a bandwidth more than $100 \mathrm{~nm}$.

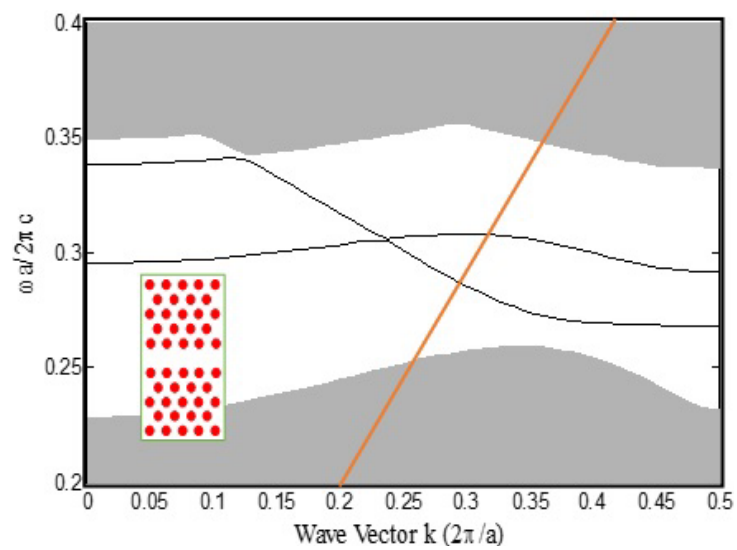

Fig. 3. The band diagram of the $\mathrm{W} 1$ waveguide used in this paper.

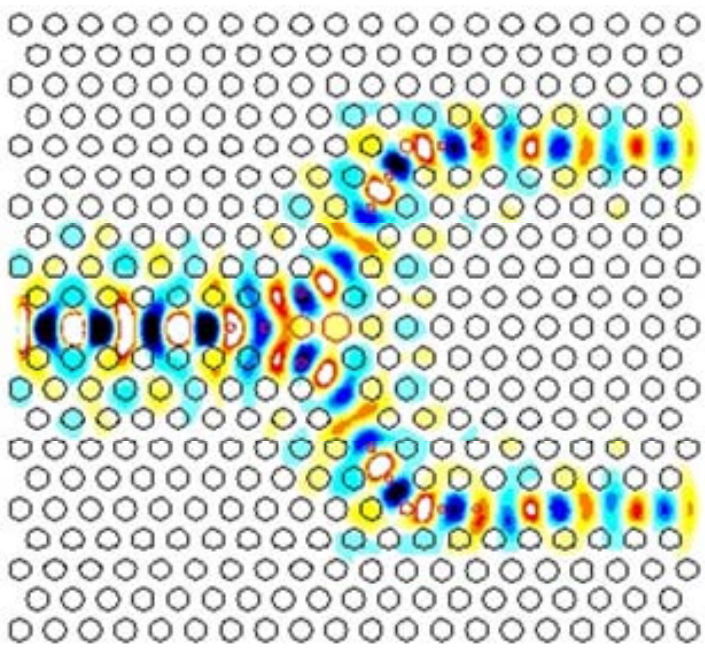

Fig. 4. The electrical field $\left(E_{Y}\right)$ distribution of the power splitter topology depicted in Fig. 2. The input light wavelength is $1550 \mathrm{~nm}$.

After simulation of the proposed structure, the transmitted power and reflected power is obtained. The bandwidth of the designed power splitter is more than $85 \mathrm{~nm}$. In addition, its transmission is about $98 \%$. The power distribution in outputs and power transmittance are shown in Fig. 4 and Fig. 5 respectively.

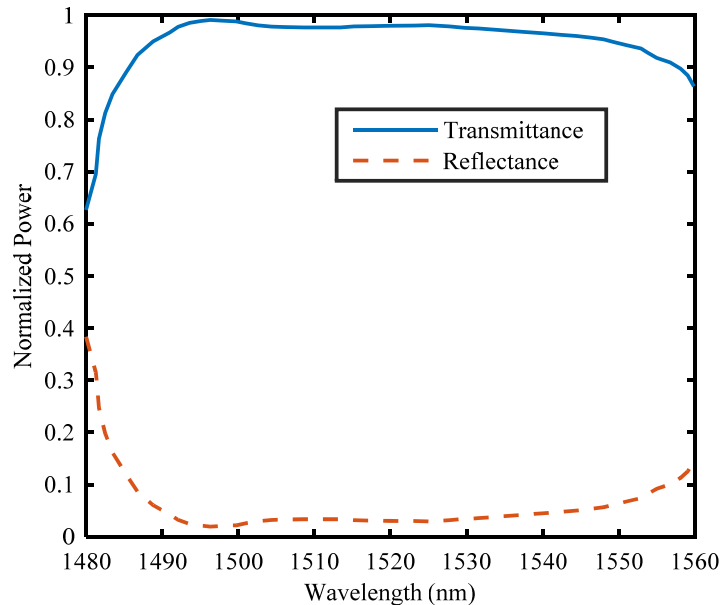

Fig. 5. The normalized transmission (summation of two output arms) and reflection for the proposed Y-splitter derived by FDTD method.

\section{Output port}

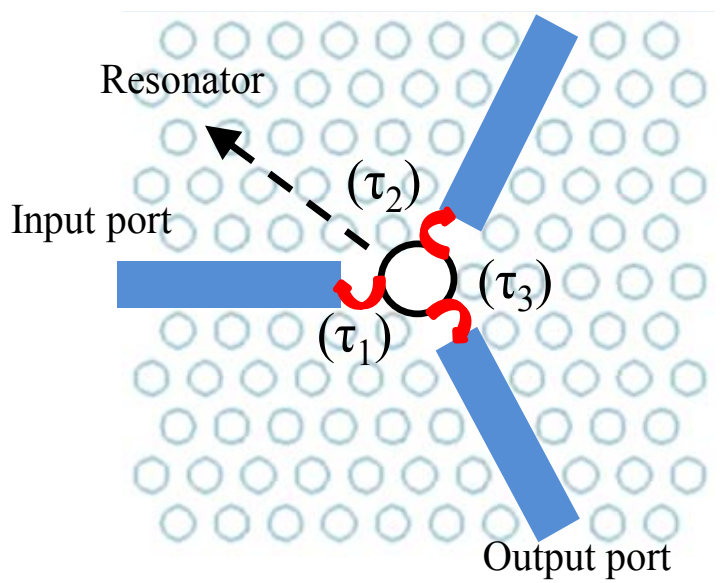

Fig. 6: Schematic model for a PhC Y-junction in a hexagonal lattice of circular holes embedded in a dielectric substrate. The radius of holes are equal to $0.3 a$.

Using the coupled mode theory approach, A $\mathrm{PhC} \mathrm{Y-junction} \mathrm{can} \mathrm{be} \mathrm{modelled} \mathrm{as} \mathrm{a} \mathrm{resonator}$ which is coupled to three waveguides [14]. As shown in Fig. 6, one of which is the input and the other two are output waveguides. The amplitude decay rate from the central resonator to each output is assumed to be $\tau_{\mathrm{i}}$. Assuming that " $\mathrm{A}$ " is the amplitude of resonance at the central cavity, Yang et al. showed that the $\mathrm{Y}$-junction can be treated as a resonator that couples with the mentioned ports, bases on the following equations: 


$$
\begin{aligned}
& \frac{d A}{d t}=j \omega_{0} A-A\left(\sum_{i} \frac{1}{\tau_{i}}\right)+\sum_{i}\left(S_{+i} \sqrt{\frac{2}{\tau i}}\right) \\
& S_{-i}=-S_{+i}+\sqrt{\frac{2}{\tau_{i}} A}
\end{aligned}
$$

Where $\mathrm{S}_{+\mathrm{i}}$ and $\mathrm{S}_{-\mathrm{i}}$ are the amplitudes of the waves travelling in the positive and negative direction of each waveguide. It is shown in [17] that using the coupled mode theory the reflection coefficient can be obtained as (3).

$$
R=\left|\frac{-j\left(\omega-\omega_{0}\right)+\frac{1}{\tau_{1}}-\frac{1}{\tau_{2}}-\frac{1}{\tau_{3}}}{j\left(\omega-\omega_{0}\right)+\frac{1}{\tau_{1}}+\frac{1}{\tau_{2}}+\frac{1}{\tau_{3}}}\right|^{2}
$$

Where, $\omega_{0}$ is the resonance frequency. A simple glance at the reflection formula shows that if equation (4) is satisfied, the reflection will become zero at $\omega=\omega_{0}$.

$$
\frac{1}{\tau_{1}}=\frac{1}{\tau_{2}}+\frac{1}{\tau_{3}}
$$

If the Y-junction is three fold symmetric, like the normal junction shown in Fig., then due to symmetry $\tau_{1}=\tau_{2}=\tau_{3}$. Therefore according to (4) the reflection will not be equal to zero. The FDTD simulation of a normal and symmetrical $\mathrm{PhC} \mathrm{Y-junction} \mathrm{confirms} \mathrm{this} \mathrm{fact} \mathrm{[14].} \mathrm{As} \mathrm{a}$ matter of fact to enhance the transmittance, in our proposed structure additional holes are added to the junction area and the radii of some holes are increased. This can increase the volume of the cavity and make the cavity mode resonant with the waveguide modes. However, since we want the output ports to have the same transmittance the junction should be symmetric along the horizontal axis. We already know that due to symmetry $\tau_{2}=\tau_{3}$; therefore the mentioned condition may be simplified as (5):

$$
\tau_{1}=\frac{1}{2} \tau_{2}=\frac{1}{2} \tau_{3}
$$

It means that the in order to minimize the reflection, the decay rate of resonance to the input ports should be twice the decay rate of resonance to the output ports. In a simple Yjunction all of the three decay rate time constants are equal. The tapered structure of the holes inserted in our proposed design, improves the coupling strength between the resonator and the input waveguide. It results in the reduction of $\tau_{1}$. If the tapering is designed so that $\tau_{1}$ is decreased to half $\tau_{2}$, then the zero reflection condition can be satisfied. Numerical optimization is used to design such a tapering. Discontinuities in the path of a waveguide are the source of reflection. When the transmitted signal from the input waveguide meets the branch section, many evanescent modes are excited. A common method to reduce backward reflection and enhance the impedance matching is to use tapering or adiabatic structures. In other words in structures with gradual change, less reflection are usually observed. Hence for the Y-branch and bends used in this design tapered structures are used. In order to find the optimum value for $r_{a}, r_{b}, r_{c}, r_{d}$. and $r_{e}$ an optimization algorithm is used.

Many heuristic algorithms such as genetic algorithm (GA), artificial bee colony (ABC), simulated annealing (SA), particle swarm optimization (PSO) etc. have been proposed in the literature which can be used for optimization problems. These algorithms use pseudo random methods to find the proper input array which minimizes a predefined cost function. In our structure the goal is to both maximize the bandwidth and transmission at the same time. In order to calculate the transmittance FDTD method has to be used. The main drawback of using FDTD is that it is very time consuming. Hence it makes using the mentioned optimization algorithms which use a large initial population very time consuming and practically inefficient. The process of calculating the cost function is so time consuming that we prefer to use the gradient-based optimization methods instead. The problem associated with these methods is that they might be trapped in the local minima. 
To alleviate the mentioned problem, instead of using a single initial point for optimization, a random set of initial values can be used. The optimization process used in this paper is as follows: As there are only five parameters, complicated optimization methods are not needed. We first guess some initial values for these parameters which is a $1 \times 5$ array, named VAR. Then the first parameter is swept with a variable step and the transmittance is calculated for each value while the other are kept constant. When the optimum value is found, the optimum value for $r_{a}$ is replaced in VAR(1) and we sweep the second variable to find the best value for $\operatorname{VAR}(2)$. These process goes on until we reach $\operatorname{VAR}(5)$. Then it is started from the beginning once again. The process is repeated until $\operatorname{VAR}(1), \operatorname{VAR}(2), \ldots$, VAR(5) remain constant. In order to minimize the change of being trapped in local minima, the process is repeated once again from a different starting point, i.e. another initial guess for input variables is made. This process is repeated until the best results are observed. The optimization flowchart is summarized in Fig. 7. To have a better understanding for the improvement of transmittance in our proposed power splitter, we may divide the structure to a $\mathrm{PhC}$ bend and a PhC Y-branch.

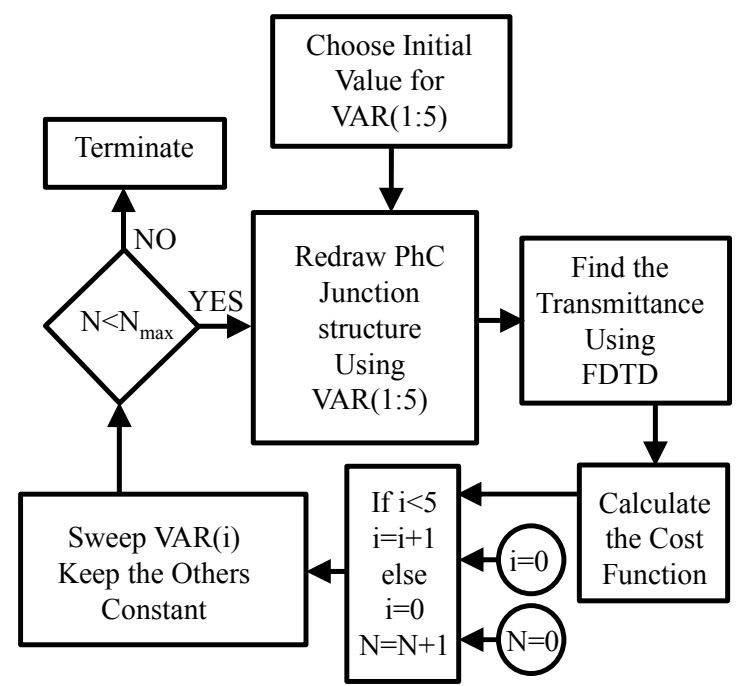

Fig. 7: The optimization flow chart.

As shown in the band diagram of the W1 waveguide in Fig. 3, the W1 waveguide which is formed by removing a row of defects supports two guiding modes. The higher frequency mode has odd parity and the lower one has odd parity. The odd mode has a bandwidth at the normalized frequency range of $0.265(a / \lambda)$ to $0.287(a / \lambda)$.

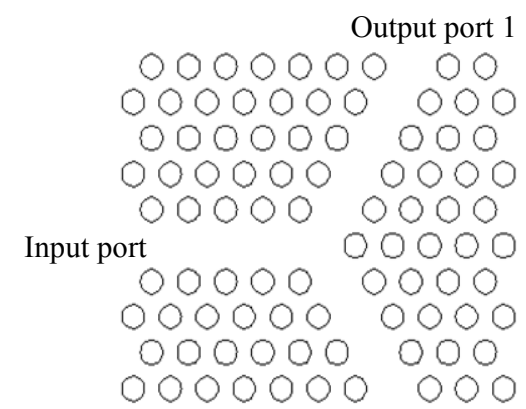

(a)

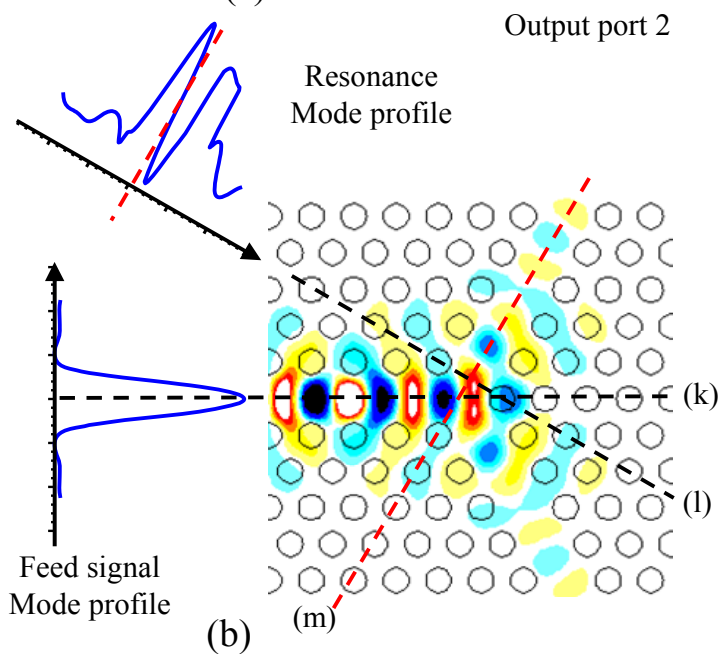

Fig. 8. (a) A simple Y-branch in a $2 \mathrm{D}$ hexagonal lattice of circular holes embedded in GaAs (b) Electrical field $\left(E_{Y}\right)$ distribution of a simple PhC Ybranch for an input wavelength of $1550 \mathrm{~nm}$.

As shown in Fig. 8, the incident wave has a Gaussian mode profile. As said before, when the input waveguide and output waveguides meet a resonator is formed. A simple $\mathrm{Y}$ Branch may be formed at the intersection of three W1 waveguides as shown in Fig. 8(a). When the input signal reaches the intersection, it starts resonating. Figure 8(b) shows the vertical component of electrical field (Ey) when the input normalized frequency is equal to $0.275(a / \lambda)$. As seen in Fig. 8(b) the incident wave has an even parity along the axis labeled " $k$ ". The resonator acts as a feed for output waveguides. If the resonator is designed in such a manner that the mode profile along the 
"l" axis is also Gaussian a good coupling will occur. We have depicted the resonance at the "l" axis for the simple Y-branch. As seen the mode profile is not even. However it can be considered as the superposition of an odd mode and an even mode. For the normalized frequency of $0.275(\mathrm{a} / \lambda)$, the $\mathrm{W} 1$ waveguide only supports the even modes. While the resonance is not purely even. Therefore poor coupling is expected. To have a better understanding of a good resonance profile, a hypothetical model is shown in Fig 9(a).

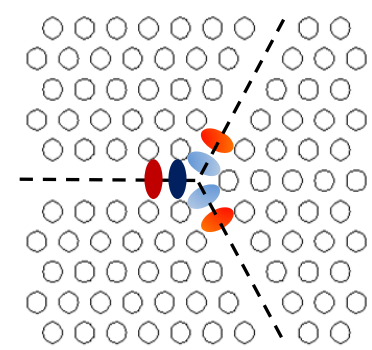

(a)

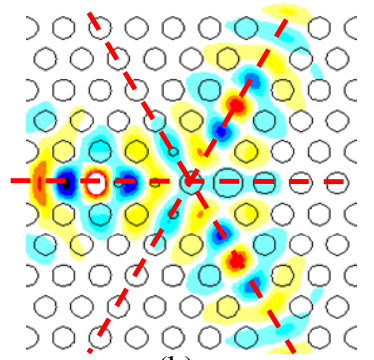

(b)
Fig. 9. The ideal resonance of a hypothetical Ybranch (b) The resonance profile obtained for the optimized Y-branch proposed in this paper for an input wavelength of $1550 \mathrm{~nm}$.

The profile of electrical field should be symmetric along the depicted axis to provide good coupling between the input and output waveguides. The resonance profile of our structure is also shown in Fig. 9(b). The threefold resonance symmetry obtained for these $\mathrm{Y}$ branch guaranties a good coupling. To explain the existence of reflection at the bends, the bending area can be assumed as another waveguide whose supercell is shown in the top section of Fig. 10. It can be assumed that the bend is comprised of two W1 waveguides which are coupled to this waveguide. PWE method has been used to find the band diagram regarding this transition section. The band diagram obtained in this case is superimposed on the original band diagram. As seen the group velocity of this modes are different. Hence a reflection is expected. It can be shown that adding $r_{e}$ and $r_{d}$ holes reduce the effective index of the transition section which leads to shifting these modes up. As a result better coupling is observed.

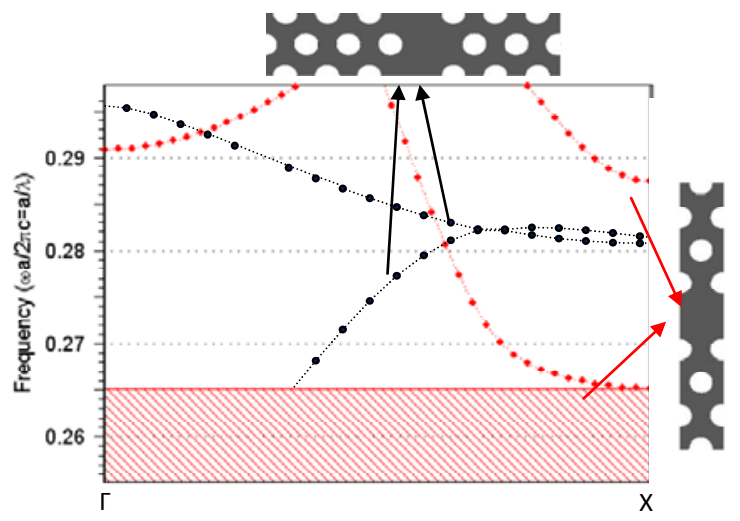

Fig. 10: The band diagram of a W1 waveguide and the band diagram of the transition section of a 60 degree bend.

\begin{tabular}{cccc}
\multicolumn{4}{c}{ TABLE 1 COMPARISON OF THE PROPOSED STRUCTURE WITH THE } \\
\multicolumn{3}{c}{ STRUCTURES PROPOSED IN THE LITERATURE } \\
\hline \hline Ref. & Year & Bandwidth & Transmission \\
\hline \hline$[20]$ & 2011 & $105 \mathrm{~nm}$ & $95 \%$ \\
{$[25]$} & 2014 & $11 \mathrm{~nm}$ & $96.3 \%$ \\
{$[26]$} & 2014 & $43 \mathrm{~nm}$ & $99 \%$ \\
{$[27]$} & 2014 & $70 \mathrm{~nm}$ & $90 \%$ \\
{$[32]$} & 2014 & $30 \mathrm{~nm}$ & $95 \%$ \\
{$[28]$} & 2014 & $111 \mathrm{~nm}$ & $96 \%$ \\
{$[29]$} & 2015 & $10 \mathrm{~nm}$ & $95 \%$ \\
{$[30]$} & 2015 & $35 \mathrm{~nm}$ & $86 \%$ \\
This work & 2016 & $85 \mathrm{~nm}$ & $98 \%$ \\
\hline
\end{tabular}

As it can be seen in Table 1 the structure proposed in this paper can provide an average $98 \%$ transmission over a $85 \mathrm{~nm}$ bandwidth which is more than all except [20] and [28]. Although the bandwidth is less but it can provide better transmission ratio than [20] and [28], both of which are simulation results.

\section{Conclusion}

A PhC Y-splitter for TE modes was proposed in this paper. FDTD method was used to analyze the proposed structure. Simulation results confirm that in comparison to the most recent structures reported in literature, the proposed Y-splitter provides an improved transmittance and bandwidth, while it has a much simpler design. For a bandwidth more than $85 \mathrm{~nm}$ the transmitted power is over than $98 \%$ and less than $2 \%$ of power is reflected to the input. 


\section{REFERENCES}

[1] P.S.J. Russell, T.A. Birks, J.C. Knight, R.F. Cregan, and J.-P. De Sandro "Silica/air photonic crystal fibres," Jap. J. Appl. Phys. vol. 37, pp. 45 - 48, 1998.

[2] J.C. Knight, T.A. Birks, P. St. J. Russell, and D.M. Atkin "All-silica single-mode optical fiber with photonic crystal cladding," Opt. Lett. vol. 21, pp. 1547-1549, 1996.

[3] J.D. Joannopoulos, P.R. Villeneuve, and S. Fan, "Photonic crystals: putting a new twist on light," Nature, vol. 386, pp. 143-149, 1997.

[4] F. Mehdizadeh and H. Alipour-Banaei, "Band gap management in two dimensional photonic crystal thue-morse structures," J. of Opt. Commun. vol. 34, pp. 61-65, 2013.

[5] D. Liu, Y. Gao, D. Gao D, and X. Han, "Photonic band gaps in two-dimensional photonic crystals of core shell-type dielectric nanorod heterostructures," Opt. Commun. vol. 285, pp. 1988-1992, 2012.

[6] L. Scolari, S. Gauza, H. Xianyu, L. Zhai, L. Eskildsen, T.T. Alkeskjold, S-T. Wu, and A. Bjarklev, "Frequency tunability of solid-core photonic crystal fibers filled with nanoparticledoped liquid crystals," Opt. Exp. vol. 17, pp. 3754-3764, 2009.

[7] M. Danaie and H. Kaatuzian, "Design of a photonic crystal differential phase comparator for a Mach-Zehnder switch," J. of Opt. vol. 13, pp. 015504, 2010.

[8] I. Park, H.-S. Lee, H.-J. Kim, K.-M. Moon, S.G. Lee, B-H. O, S-G . Park, and E-H. Lee, "Photonic crystal power-splitter based on directional coupling," Opt. Exp. vol. 12, pp. 3599-3604, 2004.

[9] S. Fan, S.G. Johnson, J. Joannopoulos, C. Manolatou, and H. Haus, "Waveguide branches in photonic crystals," J. Opt. Soc. Am. B, vol. 18, pp. 162-165, 2001.

[10]Y. Zhang, Z. Li, and B. Li, "Multimode interference effect and self-imaging principle in two-dimensional silicon photonic crystal waveguides for terahertz waves," Opt. Exp. vol. 14, pp. 2679-2689, 2006.

[11]S. Foghani, H. Kaatuzian, and M. Danaie, "Simulation and design of a wideband Tshaped photonic crystal splitter," Optica Applicata, vol. 40, pp. 863-872, 2010.
[12] N. Nozhat and N. Granpayeh, "Analysis and simulation of a photonic crystal power divider," J. of Appl. Sciences, vol. 7, pp. 35763579, 2007.

[13] A. Ghaffari, M. Djavid, and M. Abrishamian, "Bi-periodic photonic crystal Y-splitter," Physica E: Low-dimensional Systems and Nanostructures, vol. 41, pp. 1495-1499, 2009.

[14] Y.D. Yang, H. Tian, and Y. Ji, "Highbandwidth and low-loss photonic crystal power-splitter with parallel output based on the integration of Y-junction and waveguide bends," Opt. Commun. vol. 285, pp. 37523757, 2012.

[15] J.-S. Li and H. Liu, "Terahertz polarization beam splitter based on two photonic crystal cavities," Optik-International J. Light and Electron. Opt. vol. 126, pp. 139-143, 2015.

[16] M. Lin, X. Jin, Z. Ouyang, G. Zheng, and G. Wen, "Y-type polarization beam splitter based on polarization-selective defects within crystal waveguides in a square-lattice photonic crystal with solid rods," Chin. Opt. Lett. vol. 13, pp. s11301- 4, 2015.

[17] Z. Huang, X. Yang, Y. Wang, X. Meng, R. Fan, and L. Wang, "Ultrahigh extinction ratio of polarization beam splitter based on hybrid photonic crystal waveguide structures," Opt. Commun. vol. 354, pp. 9-13, 2015.

[18] H.A. Badaoui, M. Feham, and M. Abri, "Double bends and Y-shaped splitter design for integrated optics," Prog. In Electromag. Res. Lett., vol. 28, pp. 129-138, 2012.

[19]P. Khurana and S. Mishra, "Photonic Crystal Waveguide Based Y-Junction Splitter Through Finite Difference Time Domain (FDTD) Simulation Method," IJSTR, Vol. 3, pp. 186195, 2014.

[20] M. Danaie and H. Kaatuzian, "Bandwidth improvement for a photonic crystal optical Ysplitter," J. of the Opt. Soc. of Korea, vol. 15, pp. 283-288, 2011.

[21] M.B. Yucel, A. Cicek, and B. Ulug, "Polarization-independent beam splitting by a photonic crystal right prism," Appl. Phys. B, vol. 113, pp. 107-114, 2013.

[22] J. Sharma, R. Sharma, and L.K. Dusad, "Review and analysis of photonic crystal beam splitters for optical communication applications," in Proc. International Conf. on 
Green Computing and Internet of Things, India. pp. 160-162, 2015.

[23] Y. $\mathrm{Xu}$ and J. Xiao, "An ultracompact polarization-insensitive silicon-based strip-toslot power splitter," IEEE Photon. Technol. Lett. vol. 28, pp. 536-539, 2016.

[24] D.C. Tee, T. Kambayashi, S.R. Sandoghchi, N. Tamchek, and F.R.M. Adikan, "Efficient, Wide Angle, Structure Tuned $1 \times 3$ Photonic Crystal Power Splitter at $1550 \mathrm{~nm}$ for Triple Play Applications," J. Lightwave Technol. vol. 30, pp. 2818-2823, 2012.

[25]D. Tee, N. Tamchek, Y. Shee, and F.M. Adikan, "Numerical investigation on cascaded $1 \times 3$ photonic crystal power splitter based on asymmetric and symmetric $1 \times 2$ photonic crystal splitters designed with flexible structural defects," Opt. Exp. vol. 22, pp. 24241-24255, 2014.

[26] H. Wang and L. He, "Highly efficient $1 \times 3$ power splitter at $1550 \mathrm{~nm}$ for triple play applications using photonic crystal waveguides," Opt. Eng. vol. 53, pp. 075104075104, 2014.

[27] J. Zhou, H. Tian, D. Yang, Q. Liu, L. Huang, and Y. Ji, "Low-loss, efficient, wide-angle $1 \times 4$ power splitter at $\sim 1.55 \mu \mathrm{m}$ wavelengths for four play applications built with a monolithic photonic crystal slab," Appl. Opt. vol. 53, pp. 8012-8019, 2014.

[28] Z. Wang and B. Ning, "Compact, wide bandwidth, multi-channel power dividers based on one-dimensional photonic crystal waveguides," Optik, vol. 125, pp. 694-696, 2014.

[29] B. Chen, C. Liu, and J. Si, "Design of broadband power splitters using two-mode interference in slot waveguides," Opt. Commun. vol. 355, pp. 367-375, 2015.

[30] H.A. Badaoui and M. Abri, "Optimized $1 \times 8$ compact splitter based on photonic crystal using the two-dimensional finite-difference time-domain technique," Opt. Eng. vol. 54, pp. 067104-067104, 2015.

[31] K. Ren and X. Ren, "Y-shaped beam splitter by graded structure design in a photonic crystal," Chin. Sci. Bul. vol. 57, pp. 1241$1245,2012$.

[32] M.K. Moghaddam, M.M. Mirsalehi, and A.R. Attari, "Slow light transmission in a photonic crystal power splitter with parallel outputs," Photon. Nanostructures: Fundam. Appl. vol. 12, pp. 75-82, 2014.

[33] Y.B. Chen, X.M. Xu, and W. Li, "Study the coupled-cavity waveguides photonic crystal power splitter," Adv. Mater. Research, vol. 900, pp. 222-225, 2014.

[34] W. Yang, X. Chen, X. Shi, and W. Lu, "Design of a high transmission Y-junction in photonic crystal waveguides," Physica B: Conden. Matt. vol. 405, pp. 1832-1835, 2010.

[35] T. Yu, H. Zhou, Z. Gong, J. Yang, X. Jiang, and M. Wang, "Ultracompact multiway beam splitters using multiple coupled photonic crystal waveguides," J. Phys. D: Appl. Phys. vol. 41, pp. 095101, 2008.

[36] S. Fan, S.G. Johnson, J. Joannopoulos, C. Manolatou, and H. Haus, "Waveguide branches in photonic crystals," J. Opt. Soc. Am. B, vol. 18, pp. 162-165, 2001.

[37] M. Soltani, A. Haque, B. Momeni, A. Adibi, $\mathrm{Y}$. $\mathrm{Xu}$, and $\mathrm{R}$. K. Lee, "Designing complex optical filters using photonic crystal microcavitites," Integr. Optoelectron. Dev. vol. 5000, pp. 257-265, 2003.

[38] T. F. Krauss, R. M. De La Rue, and S. Brand, "Two-dimensional photonic-bandgap structures operating at near-infrared wavelengths," Nature, vol. 383, pp. 699-702, 1996.

[39] M. Lončar, D. Nedeljković, T. Doll, J. Vučković, A. Scherer, and T.P. Pearsall, "Waveguiding in planar photonic crystals," Appl. Phys. Lett. vol. 77, pp. 1937-1939, 2000.

[40] E. Chow, S.Y. Lin, S.G. Johnson, P.R. Villeneuve, J.D. Joannopoulos, J.R. Wendt, G.A. Vawter, W. Zubrzycki, H. Hou, and A. Alleman, "Three-dimensional control of light in a two-dimensional photonic crystal slab," Nature, vol. 407, pp. 983-986, 2000.

[41] M. Notomi, A. Shinya, K. Yamada, J. Takahashi, C. Takahashi, and I. Yokohama, "Single mode transmission within photonic bandgap of width-varied single-line-defect photonic crystal waveguides on SOI substrates," Electron. Lett. vol. 37, pp. 293295, 2001.

[42] H. Shou-Zhen, T. Jie, R. Cheng, X. XingSheng, L. Zhi-Yuan, C. Bing-Ying, and Z. 
Dao-Zhong, "A Y-branch photonic crystal slab waveguide with an ultrashort interport interval," Chin. Phys. Lett. vol. 22, pp. 19341936, 2005.

[43] T. Yamashita and J. Summers, "Evaluation of self-collimated beams in photonic crystal for optical interconnects," IEEE J. Select. Areas in Commun. vol. 23, pp 1341-1347, 2005.

[44] L.H. Frandsen, P.I. Borel, Y.X. Zhuang, A. Harpøth, M. Thorhauge, M. Kristensen, W. Bogaerts, P. Dumon, R. Baets, V. Wiaux, J. Wouters, and S. Beckx, "Ultralow-loss 3-dB photonic crystal waveguide splitter," Opt. Lett. vol. 29, pp. 1623-1625, 2004.

[45] P.I. Borel, L.H. Frandsen, A. Harpøth, M. Kristensen, J.S. Jensen, and O. Sigmund, "Topology optimised broadband photonic crystal Y-splitter,” Electron. Lett. vol. 41, pp. 69-71, 2005.

[46] L. Dekkiche and R. Naoum, "Improved transmission for photonic crystal Y-junctions," Electr. Eng. vol. 89, pp. 71-77, 2006.

[47] R. Wilson, T.J. Karle, I. Moerman, and T.F. Krauss, "Efficient photonic crystal Yjunctions," J. Opt. A: Pure and Appl. Opt. vol. 5, p. S76-S80, 2003.

[48] M. Koshiba, Y. Tsuji, and M. Hikari, "Timedomain beam propagation method and its application to photonic crystal circuits," J. Lightwave Technol. vol. 18, pp. 102-110, 2000.

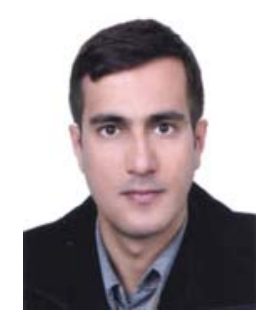

Mohammad Danaie was born in Mashhad, Iran in 1982. He received his Bachelor and Master degrees from Ferdowsi university of Mashhad in 2005 and 2007 respectively with honors and his Ph.D. degree from Amirkabir
University of Technology, Tehran, Iran in 2011, all in electrical engineering. He has been an assistant professor of electrical engineering at Semnan University, Semnan, Iran since 2012. His current research interests include: integrated all-photonic devices, photonic crystals, plasmonics and photonic devices.

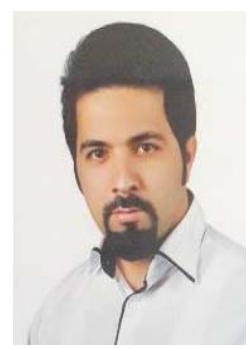

Ruhallah Nasiri Far was born in Mashhad, Iran in 1989. He received his Master degree from Hakim Sabzevari University of Sabzevar in 2015 with honors and he is keeping on his researches as a Ph.D. student in Semnan University now. His current research interests include: integrated circuit design and photonic crystals.

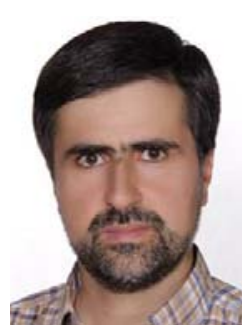

Abbas Dideban received his Ph.D. degree in Automation control from University of Grenoble I, France in 2007. He was awarded the M.Sc. degrees in Digital Electronic from Sharif University, Iran in 1997. He joined Semnan University as a lecturer in 1998. He is currently associate professor at Semnan University. His research topics include discrete event systems, Petri Nets, industrial automation, digital systems design. 